\title{
1RXS J062518.2+733433: A new intermediate polar
}

\author{
S. Araujo-Betancor ${ }^{1, \star}$, B. T. Gänsicke ${ }^{1, \star}$, H.-J. Hagen ${ }^{2}$, P. Rodriguez-Gil ${ }^{1}$, and D. Engels ${ }^{2}$ \\ 1 Department of Physics and Astronomy, University of Southampton, Southampton SO17 1BJ, UK \\ 2 Hamburger Sternwarte, Universität Hamburg, Gojenbergsweg 112, 21029 Hamburg, Germany
}

Received 1 April 2003 / Accepted 30 April 2003

\begin{abstract}
We report the identification of the cataclysmic variable 1RXS J062518.2+733433 as an intermediate polar. The orbital period of the system is determined to be $283.0 \pm 0.2 \mathrm{~min}$ from the radial velocity variation of $\mathrm{H} \alpha$, measured in an extensive set of time-resolved spectra. Differential optical photometry obtained over a base line of three weeks reveals the presence of coherent variability with a period of $19.788 \pm 0.002 \mathrm{~min}$, which we suggest to be the white dwarf spin period. The power spectrum of our photometry also contains a strong signal near the spectroscopically-determined orbital period. The emission lines in 1RXS J062518.2+733433 display a complex multicomponent structure. In the trailed spectrogram of He I $\lambda 6678$ we detect a narrow component with a radial velocity semi-amplitude of $\simeq 140 \mathrm{kms}$, consistent with a possible origin on the irradiated face of the secondary. The absence of eclipses gives an upper limit on the binary inclination of $i \lesssim 60^{\circ}$.
\end{abstract}

Key words. stars: binaries: close - stars: individual: 1RXS J062518.2+733433 - stars: novae, cataclysmic variables

\section{Introduction}

Over the past few years it has become increasingly clear that our understanding of the evolution of cataclysmic variables $(\mathrm{CVs})$ is a rather unsettled matter. A number of the predictions made by the standard theory of $\mathrm{CV}$ evolution (disrupted magnetic braking, King 1988) are in direct conflict with observations. Whereas a number of additions/alternatives to the standard model have been recently proposed (King \& Schenker 2002; Schenker \& King 2002; Schenker et al. 2002; Andronov et al. 2003), it is apparent that the disturbing disagreements between theory and observations have a common denominator: the possible impact of selection effects on the currently known population of galactic CVs. In order to quantitatively test any theory of $\mathrm{CV}$ evolution it is mandatory to establish a statistically complete sample of this class of binary stars.

There are currently a number of large-scale surveys for CVs underway that pursue this aim (e.g. Szkody et al. 2002; Marsh et al. 2002). Among these projects, our selection of CVs based on their spectroscopic properties in the Hamburg Quasar Survey (HQS; Hagen et al. 1995) has been especially prolific, resulting in the discovery of more than 50 new bright $\mathrm{CVs}$ (e.g. Gänsicke et al. 2000; Nogami et al. 2001; Gänsicke et al. 2002a,b).

Send offprint requests to: S. Araujo-Betancor,

e-mail: sab@astro.soton.ac.uk

* Visiting Astronomer, German-Spanish Astronomical Center, Calar Alto, operated by the Max-Planck-Institut für Astronomie, Heidelberg, jointly with the Spanish National Commission for Astronomy.
In this paper, we report follow-up observations of 1RXS J062518.2+733433, henceforth RX J0625, an object originally identified as a CV on the basis of its X-ray emission and optical spectrum (Wei et al. 1999). We have independently selected RX J0625 as a CV candidate because of the noticeable Balmer emission in its HQS data. The strong He II $\lambda 4686$ emission detected in the identification spectrum of RX J0625, along with coherent optical variability observed on a time scale of $\sim 20 \mathrm{~min}$, immediately reveal the intermediate polar nature of this CV. In Sect. 2 we describe the observational data and its reduction. We then derive the orbital period from the radial velocity variation of the emission lines and the spin period from differential photometry in Sect. 3. Finally, in Sect. 4 we discuss the behaviour of the strongest emission lines in detail and summarise our findings in Sect. 5.

\section{Observations and data reduction}

\subsection{Spectroscopy}

On April 292001 we obtained a single identification spectrum of RX J0625 at the Calar Alto $2.2 \mathrm{~m}$ telescope with the CAFOS focal reductor spectrograph, using the standard SITe CCD (Table 1). We used the B-200 grism and a slit width of $2^{\prime \prime}$, resulting in a useful wavelength range of 3500-7000 and a spectral resolution of $4.7 \AA$ (Fig. 1). A flux standard $\left(\mathrm{BD}+75^{\circ} 325\right)$ was observed with the same set-up in order to correct for the instrumental response. The identification spectrum contains noticeable Balmer, $\mathrm{He}_{\mathrm{I}}$ and $\mathrm{He}$ II and N/C Bowen emission lines. The strength of $\mathrm{He}$ II $\lambda 4686$ is comparable to $\mathrm{H} \beta$, indicating the presence of a strong source of ionising 




Fig. 1. Identification spectrum of RXJ0625 obtained on 2001 April 29.

Table 1. Log of observations.

\begin{tabular}{rcccc}
\hline \hline Date & UT Time & Data & Exp.(s) & Num. Obs \\
\hline Spectroscopy & & & & \\
2001 Apr. 29 & 20:37- 20:47 & B-200 & 600 & 1 \\
2002 Dec. 09 & $01: 09-01: 21$ & G-100 & 600 & 2 \\
2002 Dec. 13 & $04: 05-05: 44$ & G-100 & 600 & 9 \\
2002 Dec. 14 & $23: 20-00: 08$ & G-100 & 600 & 5 \\
2002 Dec. 15 & $03: 00-03: 45$ & G-100 & 600 & 5 \\
2002 Dec. 15 & $05: 23-06: 07$ & G-100 & 600 & 5 \\
2002 Dec. 15 & $22: 47-23: 32$ & G-100 & 600 & 5 \\
2002 Dec. 16 & $01: 39-02: 24$ & G-100 & 600 & 5 \\
2002 Dec. 28 & $23: 04-23: 26$ & G-100 & 600 & 3 \\
2002 Dec. 29 & $01: 12-01: 34$ & G-100 & 600 & 3 \\
2002 Dec. 29 & $03: 58-04: 20$ & G-100 & 600 & 3 \\
Photometry & & & & \\
2002 Dec. 09 & $01: 29-06: 23$ & V & 30 & 386 \\
2002 Dec. 15 & $00: 57-02: 14$ & Clear & 30 & 100 \\
2002 Dec. 15 & $02: 33-02: 39$ & Clear & 30 & 10 \\
2002 Dec. 16 & $00: 00-01: 15$ & Clear & 30 & 100 \\
2002 Dec. 28 & $21: 07-22: 23$ & V & 30 & 100 \\
2002 Dec. 28 & $23: 43-00: 51$ & V & 30 & 98 \\
2002 Dec. 29 & $01: 48-03: 41$ & V & 30 & 270 \\
2002 Dec. 29 & $23: 55-03: 36$ & V & 30 & 23 \\
2002 Dec. 30 & $03: 55-04: 48$ & V & 30 & 293 \\
2002 Dec. 31 & $04: 11-06: 00$ & V & 30 & 136 \\
\hline
\end{tabular}

photons in RX J0625, typical of either magnetic CVs or novalike variables. In order to determine the orbital period of RX J0625, we obtained 45 higher resolution spectra, again with CAFOS at the Calar Alto $2.2 \mathrm{~m}$ telescope (Table 1). This time the G-100 grism was used in conjuction with a $1.2^{\prime \prime}$ slit which gave a wavelength range of 4240-8300 $\AA$ and a spectral resolution of $2.1 \AA$. The higher resolution spectra were obtained over a period of 3 weeks, optimising the sampling for an efficient period determination.

All spectra were reduced in a standard manner using the Figaro package within the Starlink software collection. The frames were corrected for the bias level by subtracting
Table 2. Equivalent widths and line widths (corrected for the instrumental resolution) of the strongest emission lines measured from the average of the 45 spectra obtained in December 2002.

\begin{tabular}{lrr}
\hline \hline Line & $F W H M[\AA]$ & $E W[\AA]$ \\
\hline $\mathrm{H} \gamma$ & $13.7 \pm 0.2$ & $14.3 \pm 1.0$ \\
$\mathrm{He}_{\mathrm{II}} \lambda 4686$ & $14.2 \pm 0.3$ & $15.0 \pm 2.0$ \\
$\mathrm{H} \beta$ & $14.2 \pm 0.2$ & $22.0 \pm 2.0$ \\
$\mathrm{He}_{\mathrm{I}} \lambda 5876$ & $14.0 \pm 0.3$ & $9.6 \pm 0.5$ \\
$\mathrm{H} \alpha$ & $15.8 \pm 0.2$ & $64.0 \pm 2.0$ \\
$\mathrm{He}$ I $\lambda 6678$ & $17.3 \pm 0.2$ & $7.5 \pm 0.5$ \\
\hline
\end{tabular}

the mean of a series of bias images taken at the start and end of each observing night. Dome flat-fields were used to remove pixel to pixel variations of the chip. The spectra were then optimally extracted (Horne 1986) and sky line subtracted using Tom Marsh's Pamela package. Especial care was taken to account for the tilt of the spectra in order to maximise the signal-to-noise ratio. The wavelength calibration was performed using mercury-cadmium, helium-argon and rubidium arcs. Uncertainties on every data point calculated from photon statistics are rigorously propagated through every stage of the data reduction. We did not attempt to flux-calibrate the higher resolution spectra as it was not required for the radial velocity analysis described below.

Table 2 gives the equivalent widths and line widths of the main emission lines detected in the average of the 45 high resolution spectra.

\subsection{Photometry}

We obtained differential $V$-band and filter-less photometry of RX J0625 during 6 nights in December 2002 with the CAFOS SITe CCD camera on the Calar Alto $2.2 \mathrm{~m}$ telescope (Table 1). In order to achieve a high time resolution $(\sim 30 \mathrm{~s})$, only a small window of the chip $\left(\sim 3^{\prime} \times 2^{\prime}\right)$ was read out. The data were bias-subtracted and flat-fielded in a standard fashion using the ESO-MIDAS package, and aperture magnitudes were extracted with the sextractor (Bertin \& Arnouts 1996). The $V$ magnitudes of RX J0625 were derived relative to the HST Guide Star GSC $0437000234(V=13.4 \pm 0.4)$, located 1'50" southwards of RX J0625. This comparison star was saturated in a number of the filter-less CCD images obtained on December $14 / 15$, and, hence, we used GSC $0437000998(V=14.9 \pm 0.4)$, located $1^{\prime} 10^{\prime \prime}$ northwards of RX J0625 for the reduction of these images. RX J0625 was found at an average magnitude of $14.80 \pm 0.05$ throughout the nights in which we used the $V$ band filter. The light curves of RX J0625 clearly reveal the presence of variability with a period of $\sim 20 \mathrm{~min}$ and an amplitude of $\sim \pm 0.2$ mag throughout all nights (Fig. 2). In addition to this short-term variation the light curve of RX J0625 displays a modulation on time scales of several hours. 


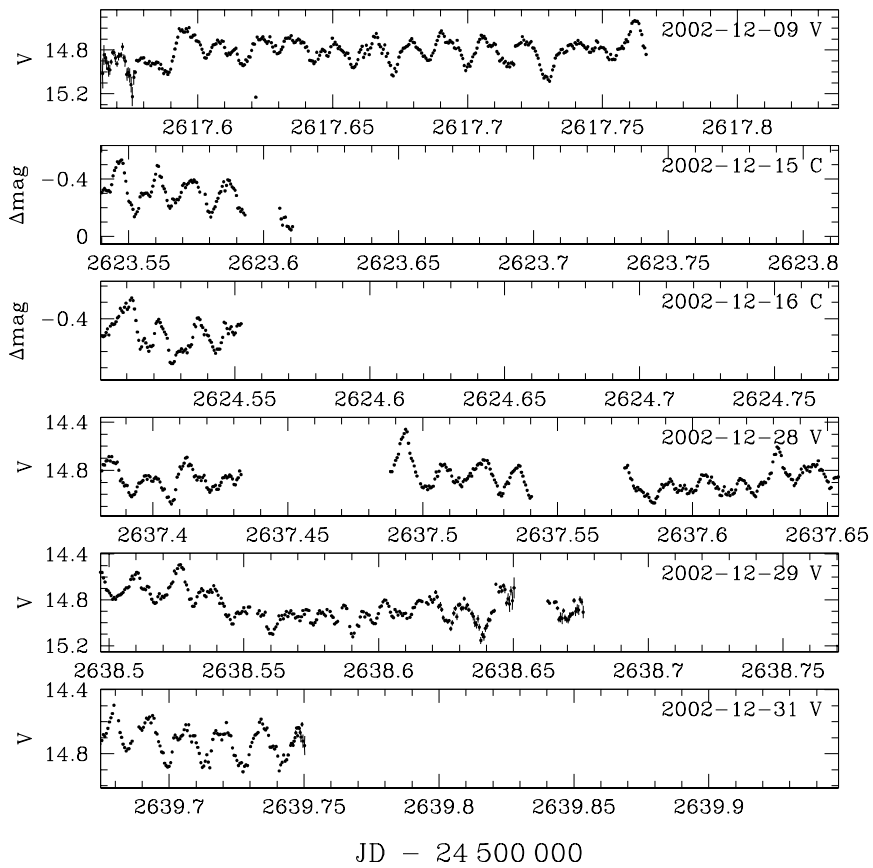

Fig. 2. Differential CCD $V$-band (V) and filter-less (C) photometry obtained at the Calar Alto $2.2 \mathrm{~m}$ observatory. Note the different scales of the filter-less and $V$ band data.

\section{Analysis}

\subsection{Spectroscopy}

The primary aim of our time-resolved spectroscopy of RX J0625 is to measure its orbital period from the radial velocity variations of the emission lines. The line profiles clearly display a complicated multicomponent structure (Fig. 3). In a first attempt, we used a single Gaussian least square fitting procedure to determine the radial velocity variations of $\mathrm{H} \alpha, \mathrm{H} \beta$ and $\mathrm{He}$ II $\lambda$ 4686. The radial velocity data were then subjected to the following period analysis methods: (a) the phase dispersion method (Stellingwerf 1978), (b) the analysis of variance (Schwarzenberg-Czerny 1989, as implemented in the MIDAS context TSA), (c) and a sine wave $\chi^{2}$ fitting procedure, using chisq in the Starlink package period. The resulting periodograms for $\mathrm{H} \alpha$ are shown in Fig. 4. The orbital frequency (period) is inferred from the three independent techniques in a consistent way to be $\Omega=5.088 \pm 0.003 \mathrm{~d}^{-1}$ $\left(P_{\text {orb }}=283.0 \pm 0.2 \mathrm{~min}\right.$ ), where the error is computed from the $\chi^{2}$ sine wave fitting $(2 \sigma, 4$ interesting parameter). A slightly more conservative error estimate based on the results obtained from all three methods gives $P_{\text {orb }}=283.0 \pm 0.3 \mathrm{~min}$. The analysis of $\mathrm{H} \beta$ and He II $\lambda 4686$ provides consistent results for $P_{\text {orb }}$, but with significantly larger errors. The robustness of our period determination is shown in Fig. 5 were we folded the $\mathrm{H} \alpha$ radial velocities over the orbital period. The radial velocity measurements from this fit are provided in Table 3.

For comparison purposes we measured the radial velocities of $\mathrm{H} \alpha$ cross-correlating the observed line profile with a single Gaussian of fixed width of $300 \mathrm{~km} \mathrm{~s}^{-1}$ (Schneider \& Young 1980). The resulting orbital period is identical to that derived

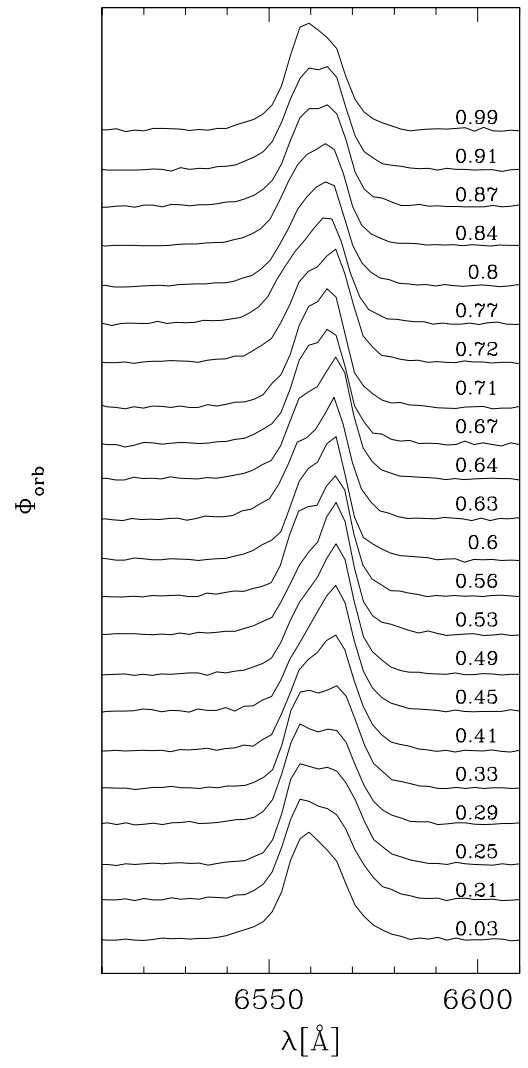

Fig. 3. Continuum-subtracted and normalised $\mathrm{H} \alpha$ profile sample sorted by orbital phases computed from Eq. (2).

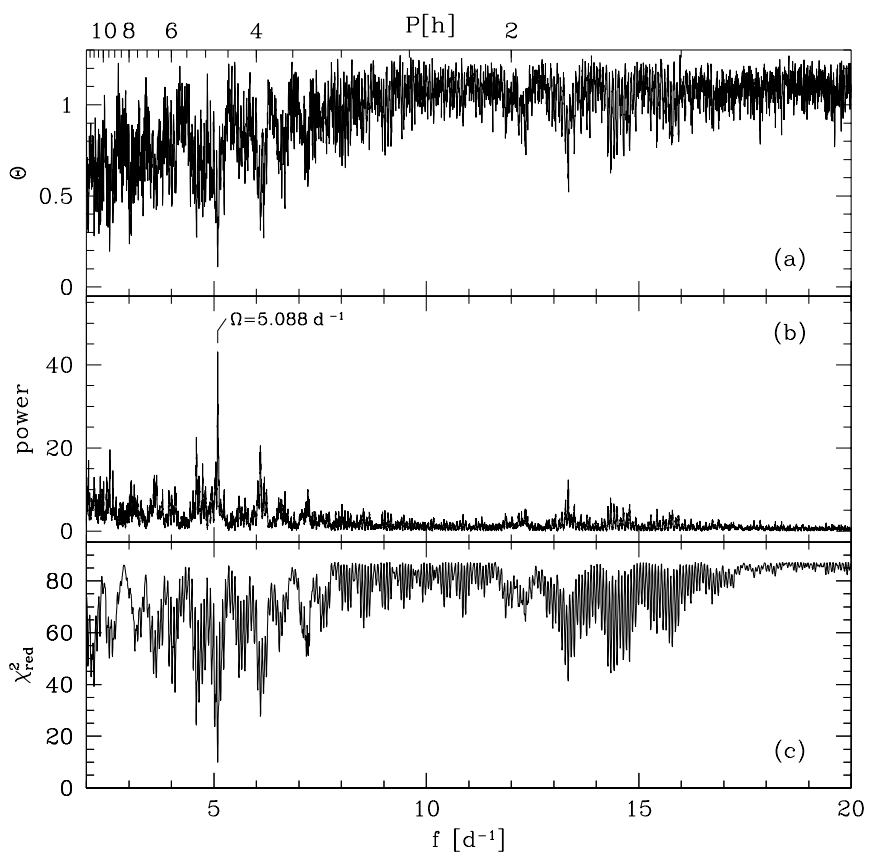

Fig. 4. Period analysis of the radial velocities measured from a Gaussfit to the $\mathrm{H} \alpha$ emission line profiles. a) Phase dispersion method periodogram. b) Analysis of variance periodogram. c) $\chi^{2}$ sine fit.

from the single Gaussian fit to the $\mathrm{H} \alpha$ profiles, but the radial velocity variation displays an amplitude larger by a factor of $\sim 2$. 
Table 3. Radial velocities of the $\mathrm{H} \alpha$ emission line determined from a single-Gaussian fit to the line profile (corrected for the heliocentric velocity).

\begin{tabular}{lcclccccc}
\hline \hline $\begin{array}{l}\text { HJD } \\
-2450000\end{array}$ & $\begin{array}{c}R V \\
{\left[\mathrm{~km} \mathrm{~s}^{-1}\right]}\end{array}$ & $\begin{array}{c}R V \text { error } \\
{\left[\mathrm{km} \mathrm{s}^{-1}\right]}\end{array}$ & $\begin{array}{l}\text { HJD } \\
-2450000\end{array}$ & $\begin{array}{c}R V \\
{\left[\mathrm{~km} \mathrm{~s}^{-1}\right]}\end{array}$ & $\begin{array}{l}R V \text { error } \\
{\left[\mathrm{km} \mathrm{s}^{-1}\right]}\end{array}$ & $\begin{array}{l}\text { HJD } \\
-2450000\end{array}$ & $\begin{array}{c}R V \\
{\left[\mathrm{~km} \mathrm{~s}^{-1}\right]}\end{array}$ & $\begin{array}{r}R V \text { error } \\
{\left[\mathrm{km} \mathrm{s}^{-1}\right]}\end{array}$ \\
\hline 617.5515 & 42.7 & 9.3 & 623.5093 & -31.0 & 6.0 & 624.4842 & 1.6 & 6.0 \\
617.5598 & 61.9 & 8.5 & 623.6290 & 24.3 & 4.8 & 624.5729 & -54.7 & 5.5 \\
621.6740 & 48.7 & 5.3 & 623.6368 & 42.1 & 4.2 & 624.5807 & -51.5 & 5.4 \\
621.6847 & 35.4 & 5.9 & 623.6446 & 64.0 & 5.0 & 624.5884 & -16.3 & 6.4 \\
621.6925 & 27.2 & 6.0 & 623.6524 & 65.4 & 6.0 & 624.5962 & -21.8 & 6.3 \\
621.7003 & 24.9 & 6.5 & 623.6601 & 64.9 & 7.1 & 624.6040 & 11.6 & 6.3 \\
621.7085 & 14.4 & 6.2 & 623.7279 & -48.5 & 4.2 & 637.4651 & -51.3 & 5.7 \\
621.7162 & 5.2 & 5.1 & 623.7357 & -61.2 & 3.8 & 637.4729 & -82.4 & 5.7 \\
621.7240 & -9.8 & 4.8 & 623.7434 & -88.2 & 3.9 & 637.4807 & -85.1 & 4.5 \\
621.7318 & -12.1 & 4.6 & 623.7512 & -84.6 & 3.8 & 637.5538 & -69.1 & 4.1 \\
621.7425 & -20.8 & 5.4 & 623.7590 & -88.2 & 4.0 & 637.5616 & -33.9 & 4.7 \\
623.4760 & 8.3 & 7.6 & 624.4531 & 26.7 & 7.2 & 637.5694 & -28.9 & 4.5 \\
623.4838 & 6.9 & 7.4 & 624.4609 & 26.7 & 7.0 & 637.6690 & -45.4 & 4.5 \\
623.4915 & 6.0 & 5.6 & 624.4687 & 31.7 & 7.0 & 637.6768 & -61.9 & 4.7 \\
623.5007 & -10.9 & 6.1 & 624.4764 & 18.9 & 6.2 & 637.6846 & -66.9 & 3.9 \\
\hline
\end{tabular}

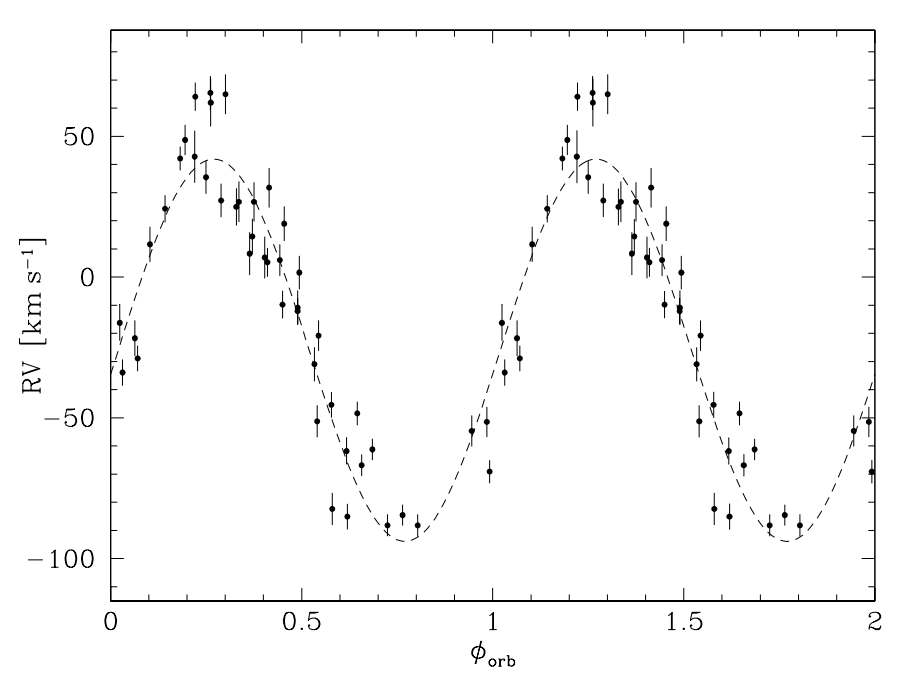

Fig. 5. Radial velocities measured from a Gauss-fit to the $\mathrm{H} \alpha$ emission line profiles, folded with the orbital period $P_{\text {orb }}=283.0 \mathrm{~min}$, using Eq. (2). The dashed curve is the best sine fit to the radial velocities.

Finally, we attempted to fit the $\mathrm{H} \alpha$ emission with a blend of a narrow and a broad Gaussian component to model more accurately the complex structure of the observed profiles. Unfortunately, this procedure did not provide unambiguous results because of the limited spectral resolution of our data. Due to the multicomponent structure of the lines profile (see Fig. 3) it is difficult to interpret the radial velocity variation obtained from either the single Gaussian fit (Fig. 5) or the Gauss correlation to $\mathrm{H} \alpha$. The behaviour of the most important emission lines is discussed in more detail in Sect. 4 on the basis of trailed spectrograms.

\subsection{Photometry}

In order to analyse the periodicities observed in the light curve of RXJ0625 (Fig.2), we have computed from the entire photometric data set both a Scargle (1982) periodogram (as implemented in the MIDAS context TSA) as well as a periodogram using the Phase Dispersion Method (PDM) of Stellingwerf (1978). Because the lengths of our individual photometric observations are of the order of or shorter than the orbital period, subtracting the nightly mean from the data would introduce erroneous signals in the low-frequency range of the periodograms. Considering that RX J0625 does not exhibit noticeable night-to-night variability, we decided to subtract from the $V$ filter and white light data the mean of all measurements obtained in the corresponding band prior to the computation of the periodograms. The resulting periodograms (Figs. 6 and 7) contain strong signals concentrated in the frequency ranges $f \lessgtr 10 \mathrm{~d}^{-1}$ and $f \simeq 60-70 \mathrm{~d}^{-1}$. The nature of the signals detected in these two separate frequency regimes is discussed below.

High-frequency signal. Figure 7 shows an enlargement around the peak signal contained in the high-frequency range of the periodogram shown in Fig. 6. Both period analysis methods consistently detect the maximum power at $72.772 \pm 0.008 \mathrm{~d}^{-1}$, corresponding to a period of $19.788 \pm 0.002 \mathrm{~min}$, whose error is given by $1 \sigma$ width of the Gaussian fit to the frequency peak. In order to test the significance of this signal, we have created a faked data set from a sine wave with a period of $19.788 \mathrm{~min}$, a similar amplitude to the observed short-period variability, and sampled exactly in the same way as the observed data. In addition, we added a $10 \%$ error to these synthetic data. The periodograms for this faked data set are plotted in gray in Fig. 7, and reproduce very well the entire structure of aliases contained in the periodograms of the observed data. We conclude from this comparison that the frequency of the maximum signal detected in the periodograms is indeed the true period underlying the short-term variability observed in RX J0625.

Coherent variability on time scales of a several minutes to several tens of minutes has been detected in the optical light 


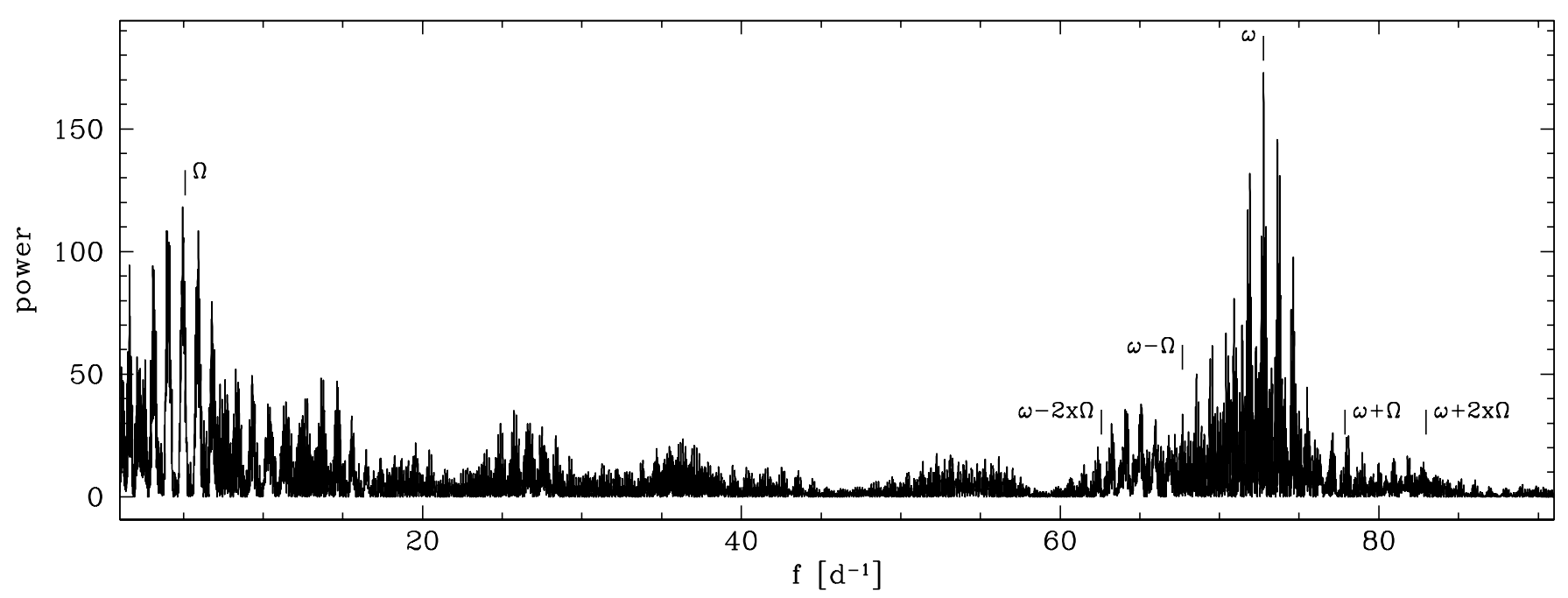

Fig. 6. Scargle periodogram of the differential CCD photometry shown in Fig. 2. $\omega$ and $\Omega$ are the white dwarf spin and orbital frequency, respectively.

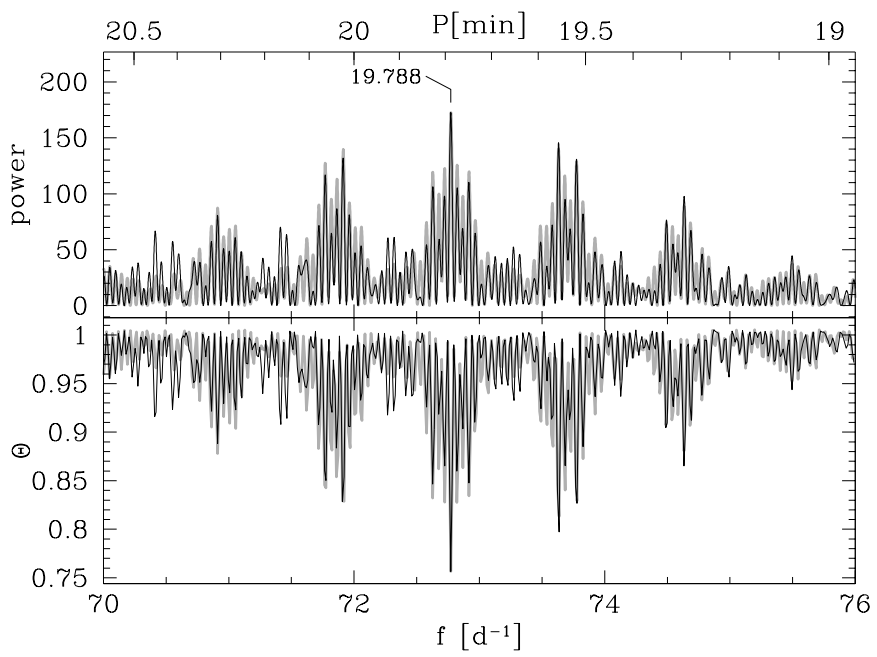

Fig. 7. Period analysis near the presumed white dwarf spin. Top panel: Scargle periodogram. Bottom panel: phase dispersion periodogram. The black lines are computed from the observed data. The gray lines are computed from a sinusoid assuming a period of $1 / \omega=19.788 \mathrm{~min}$ and identical sampling as in the observed data.

curves of a number of intermediate polars (e.g. Patterson 1994), and is interpreted as the spin period of the accreting magnetic white dwarf. We conclude from the spectral appearance of RX J0625 and from the detected coherent optical variability that RXJ0625 is indeed a new member of the small class of intermediate polars. We suggest that the detected frequency (period) of $72.772 \mathrm{~d}^{-1}$ (19.788 $\mathrm{min}$ ) is the white dwarf spin frequency $\omega$ (period $\left.P_{\text {spin }}=1 / \omega\right)$. From the combined $V$ band and filter-less photometry, we derive the following spin ephemeris:

$\phi_{0}^{\text {spin }}=$ HJD 2452617.0645(4) $+0.013742(3) \times E$

where $\phi^{\text {spin }}=0$ is defined as the spin pulse maximum. Errors in the last digit are given in brackets. Figure 8a shows our photometric data folded with this ephemeris. The quasi-sinusoidal shape of this light curve, which is typical for the spin light

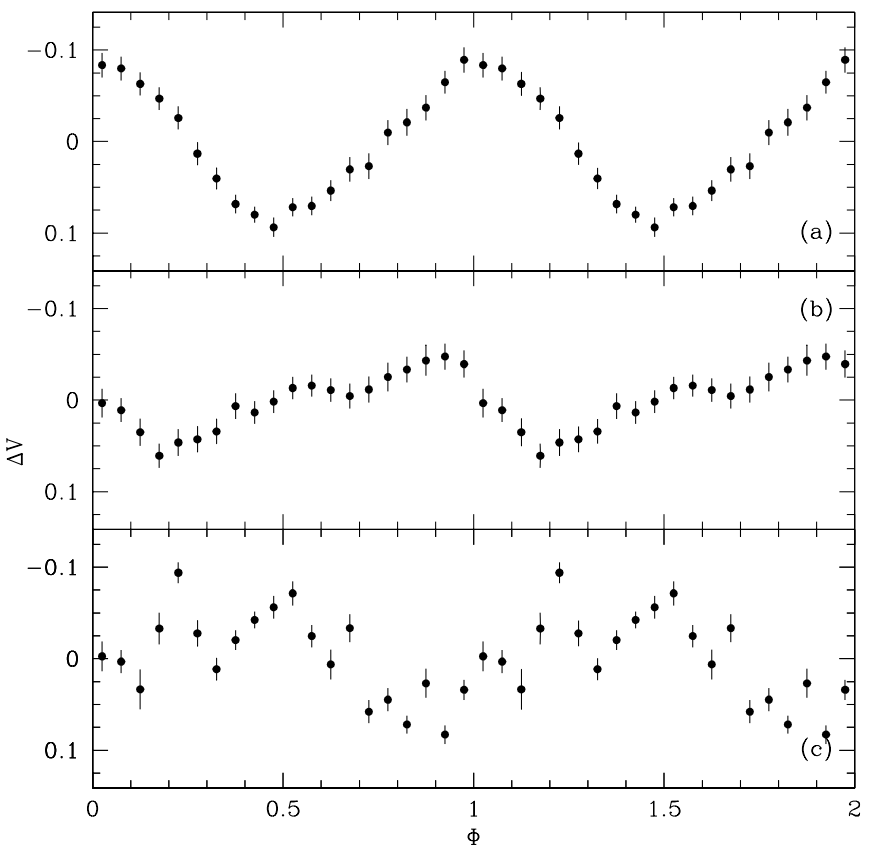

Fig. 8. The photometric data points from Fig. 2 mean-subtracted and folded over: a) The spin period of $19.788 \mathrm{~min}$, using Eq. (1). b) The beat period of $21.275 \mathrm{~min}$. c) The orbital period of $283.0 \mathrm{~min}$, using Eq. (2).

curve of intermediate polars (see, e.g., the spin light curve of FO Aqr by de Martino et al. 1994), lends support to our hypothesis that the $72.772 \mathrm{~d}^{-1}$ frequency detected in the power spectrum of RX J0625 is indeed the spin frequency of the white dwarf.

Another hallmark of intermediate polars is the detection of beat frequencies between the white dwarf spin frequency $\omega$ and the orbital frequency $\Omega$, which arise from the reprocession of X-rays emitted from close to white dwarf surface on, e.g., the secondary star. Such sideband signals have been detected in different systems at $\omega-2 \Omega, \omega-\Omega, \omega+\Omega, \omega+2 \Omega$ (see Warner 1986 for an interpretation of these frequencies). In RX J0625, 


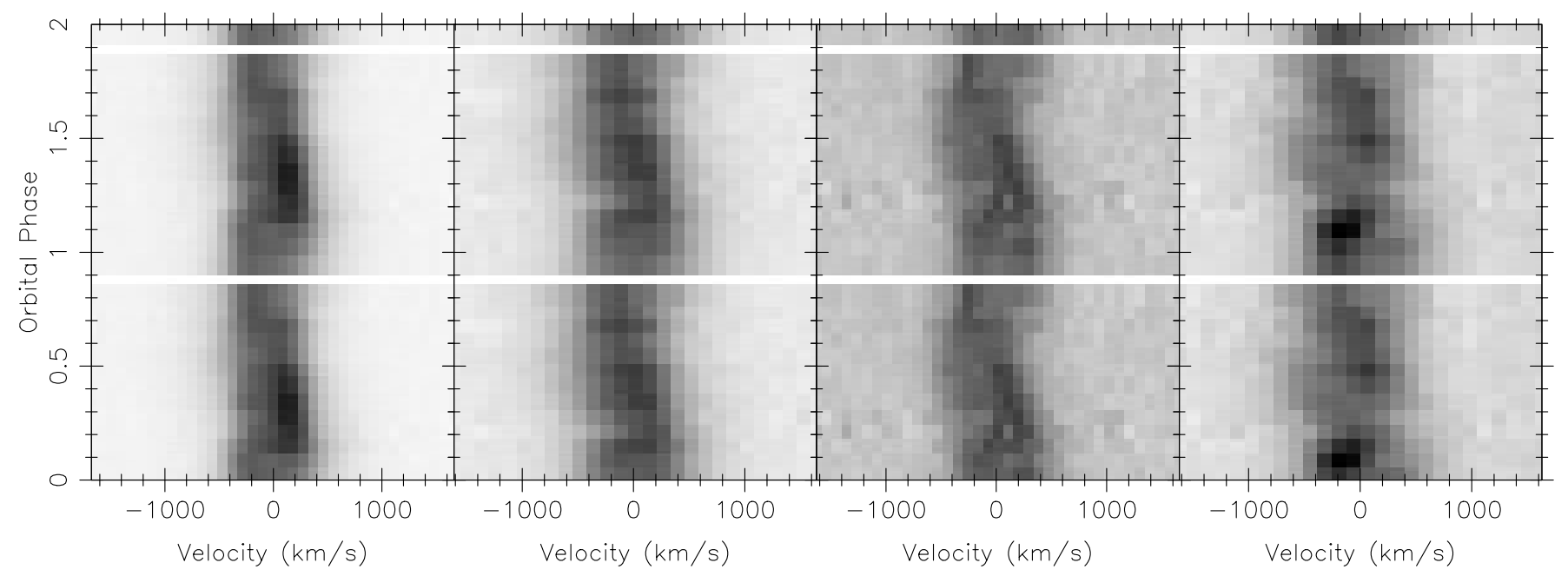

Fig. 9. Trailed spectrograms of $\mathrm{H} \alpha, \mathrm{H} \beta$, He I $\lambda$ 6678, He II $\lambda$ 4686, computed from the 45 spectra obtained in December 2002 (Table 1). Orbital phases have been computed using Eq. (2).

the only unambiguous detection of a sideband signal is $\omega-\Omega$ (see Fig. 6). The photometric data folded over this beat period of 21.275 min are shown in Fig. 8b. While we are confident that our identifications of the spin and beat period are correct we mention as a note of caution that time-resolved X-ray and/or polarimetric data are necessary to unambigously confirm this interpretation.

Low-frequency signal. The strongest signal in the lowfrequency range does not coincide with the orbital frequency derived in Sect. 3.1 from the radial velocity variation of $\mathrm{H} \alpha$, but is found at $4.944 \mathrm{~d}^{-1}$, corresponding to a period of $291.3 \mathrm{~min}$. This period is $\simeq 3 \%$ longer than the orbital period. Considering the sampling of our photometric time series, especially the fact that none of our data sets covers significantly more than one binary orbit, the most likely hypothesis is that the detected lowfrequency signal is related to the orbital motion of the binary. Further observations would be useful to test in detail whether the low-frequency photometric frequency is indeed identical to the orbital frequency $\Omega$.

\section{Discussion}

As already mentioned in Sect. 3.1, the line profiles clearly display a multicomponent structure (Fig. 3), which makes the interpretation of the determined radial velocity variations rather ambiguous (Fig. 5). To explore the behaviour of the emission lines in more detail, we constructed trailed spectra of $\mathrm{H} \alpha, \mathrm{H} \beta$, Не г $\lambda 6678$, and He II $\lambda$ 4686, which are shown in Fig. 9. These diagrams were computed from the continuum-normalised spectra after binning into 15 phase intervals. The He $\mathrm{s} \lambda 6678$ line clearly shows two emission components: a narrow one with a radial velocity semi-amplitude of $\approx 140 \mathrm{~km} \mathrm{~s}^{-1}$ and a wider one with velocity reaching $\sim \pm 500 \mathrm{~km} \mathrm{~s}^{-1}$. Both components are not in phase, the phase offset being $\sim 0.15$. The trailed spectra shown in Fig. 9 were phased according to the following orbital ephemeris

$\phi_{0}^{\text {orb }}=\operatorname{HJD} 2452617.508(5)+0.1965(1) \times E$

where the instant of zero phase was derived as the time of blueto-red crossing of the narrow component of $\mathrm{He}_{\mathrm{I}} \lambda 6678$.

In analogy to the narrow emission lines observed in many magnetic $\mathrm{CVs}$, this narrow emission line component may have its origin on the irradiated face of the secondary star. In order to test this hypothesis, we performed the following simple calculation. The secondary mass was estimated from the massperiod relation derived by Smith \& Dhillon (1998), which gives a value of $M_{2} \approx 0.48 M_{\odot}$. For the primary mass we adopted $M_{1}=0.8 M_{\odot}$ (the average white dwarf mass in long-period CVs determined by Smith \& Dhillon 1998), resulting in a mass ratio $q=M_{2} / M_{1} \approx 0.6$. Assuming a disc radius of $0.8 R_{\mathrm{L}_{1}}$, the lack of eclipses in the optical light curve of RX J0625 sets an upper limit on the orbital inclination of $i \lesssim 60^{\circ}$. The projected velocity of the secondary is given by:

$K_{2}=\frac{2 \pi a \sin i}{P_{\mathrm{orb}}(1+q)}$

where $a$ is the binary separation. With the above assumptions, the radial velocity variation of the secondary star can be as large as $K_{2} \approx 130 \mathrm{~km} \mathrm{~s}^{-1}$, which is comparable to the value obtained from the trailed spectrogram of the narrow $\mathrm{He}$ i $\lambda 6678$ line. The predicted $K_{1}$ velocity is then $\approx 80 \mathrm{~km} \mathrm{~s}^{-1}$, somewhat larger than the semi-amplitude of the radial velocity curve of the broad component of $\mathrm{H} \alpha$ (Fig. 9, see also Fig. 5), but not inconsistent.

Taken at face value, the behaviour of the narrow emission line components detected in our (admittedly rather low resolution) phase-resolved spectroscopy are consistent with an origin on the irradiated face of the secondary.

As we mentioned above, the broad component of the He I $\lambda 6678$ line displays a phase offset of $\sim 0.15$ with respect to the narrow one, reaching a velocity of $\sim-500 \mathrm{~km} \mathrm{~s}^{-1}$ at its maximum excursion to the blue. The phasing with respect to 
the narrow component (and assuming that our above interpretation of the origin of the narrow component is correct) indicates that the broad emission is not coming from an axisymmetric structure around the white dwarf. Nevertheless, the high velocity dispersion observed in the broad component clearly points towards an origin close to the white dwarf. The maximum velocity to the blue is reached at phase $\sim 0.4-0.5$. A similar high-velocity S-wave has been observed in the intermediate polars EX Hya (Hellier et al. 1989) and V1025 Cen (Hellier et al. 2002). The same components are also characteristic of the SW Sextantis stars, which have been recently proposed to be magnetic systems (Rodríguez-Gil et al. 2001). In all these systems, it is believed that these high-velocity S-waves form in the vicinity of the primary's magnetosphere.

The trailed spectra of He II $\lambda 4686$ seem to exhibit the same two emission components, with the narrow one also dominating and the same velocity amplitudes as in $\mathrm{He}_{\mathrm{I}} \lambda$ 6678. Also the trailed spectra of $\mathrm{H} \alpha$ and $\mathrm{H} \beta$ clearly show a multicomponent structure, even though their narrow components are less obvious than in Нег $\lambda 6678$. In order to securely identify the different line components with physical emission sites in the binary, it will be necessary to obtain time-resolved spectroscopy spanning several consecutive binary orbits with better spectral resolution.

\section{Conclusions}

We have observed RXJ0625 as part of our ongoing search for new CVs selected on the basis of their spectroscopic properties from the Hamburg Quasar Survey. From the radial velocity variations measured in $\mathrm{H} \alpha$, we determined an orbital period of $283.0 \pm 0.2 \mathrm{~min}$. The detection of coherent optical variations clearly classifies RX J0625 as a member of the small class of intermediate polars. The period of these variations is $19.788 \pm 0.002 \mathrm{~min}$ which is most likely the spin period of the white dwarf. In terms of its orbital and spin period, RX J0625 is very similar to FO $\operatorname{Aqr}\left(P_{\text {orb }}=291 \mathrm{~min}\right.$, and $\left.P_{\text {spin }}=21 \mathrm{~min}\right)$. Our phase-resolved spectroscopy clearly shows that the emission lines are multicomponent, and we identify a narrow component with a radial velocity semi-amplitude of $\simeq 140 \mathrm{kms}$, which might originate from the irradiated face of the secondary. Extensive photometric monitoring, phase-resolved high-resolution spectroscopy and pointed X-ray observations of RX J0625 are strongly encouraged.

Note added in proof: After submission of this paper, we became aware of an independent photometric study of RX J0625 by Staude et al. (2003) that confirms the likely white dwarf spin period and shows that the low-frequency photometric signal detected in RX J0625 is consistent with our spectroscopic orbital period within the errors of the measurements. Furthermore, the referee drew our attention to the fact that an orbital period for RX J0625 was included on December 132002 in the online catalogue of Downes et al. (2001), which is consistent with our value.
Acknowledgements. SAB thanks PPARC for a studentship. BTG was supported by a PPARC Advanced Fellowship, the HQS was supported by the Deutsche Forschungsgemeinschaft through grants Re 353/11 and Re 353/22. We thank Ana Guijarro for obtaining part of the Calar Alto observations. We are grateful to Christian Knigge for suggesting the use of chisq in the Starlink period package for the analysis of the radial velocity data - and for pointing out that the latest version of period does not work properly. Tom Marsh is thanked for providing his reduction and analysis packages. Patrick Woudt is acknowldedged for his prompt referee report and for a number of useful suggestions.

\section{References}

Andronov, N., Pinsonneault, M., \& Sills, A. 2003, ApJ, 582, 358 Bertin, E., \& Arnouts, S. 1996, A\&AS, 117, 393

de Martino, D., Buckley, D. A. H., Mouchet, M., \& Mukai, K. 1994, A\&A, 284, 125

Downes, R. A., Webbink, R. F., Shara, M. M., et al. 2001, PASP, 113, 764

Gänsicke, B. T., Fried, R. E., Hagen, H.-J., et al. 2000, A\&A, 356, L79

Gänsicke, B. T., Hagen, H. J., \& Engels, D. 2002a, On the space density of cataclysmic variables, ed. B. T. Gänsicke, K. Beuermann, \& K. Reinsch, ASP Conf. Ser., 261, 190

Gänsicke, B. T., Hagen, H.-J., Kube, J., et al. 2002b, HS 0455+8315: A new eclipsing novalike variable, ed. B. T. Gänsicke, K. Beuermann, \& K. Reinsch, ASP Conf. Ser., 261, 623

Hagen, H. J., Groote, D., Engels, D., \& Reimers, D. 1995, A\&AS, 111,195

Hellier, C., Mason, K. O., Smale, A. P., et al. 1989, MNRAS, 238, 1107

Hellier, C., Wynn, G. A., \& Buckley, D. A. H. 2002, MNRAS, 333, 84

Horne, K. 1986, PASP, 98, 609

King, A. R. 1988, QJRAS, 29, 1

King, A. R. \& Schenker, K. 2002, in The Physics of Cataclysmic Variables and Related Objects, ed. B. T. Gänsicke, K. Beuermann, \& K. Reinsch, ASP Conf. Ser., 261, 233

Marsh, T. R., Morales-Rueda, L., Steeghs, D., et al. 2002, in The Physics of Cataclysmic Variables and Related Objects, ed. B. T. Gänsicke, K. Beuermann, \& K. Reinsch, ASP Conf. Ser., 261, 200 Nogami, D., Kato, T., Baba, H., et al. 2001, MNRAS, 322, 79

Patterson, J. 1994, PASP, 106, 209

Rodríguez-Gil, P., Casares, J., Martínez-Pais, I. G., Hakala, P., \& Steeghs, D. 2001, ApJ, 548, L49

Scargle, J. D. 1982, ApJ, 263, 835

Schenker, K., \& King, A. R. 2002, in The Physics of Cataclysmic Variables and Related Objects, ed. B. T. Gänsicke, K. Beuermann, \& K. Reinsch, ASP Conf. Ser., 261, 242

Schenker, K., King, A. R., Kolb, U., Wynn, G. A., \& Zhang, Z. 2002, MNRAS, 337, 1105

Schneider, D. P., \& Young, P. 1980, ApJ, 238, 946

Schwarzenberg-Czerny, A. 1989, MNRAS, 241, 153

Smith, D. A., \& Dhillon, V. S. 1998, MNRAS, 301, 767

Staude, A., Schwope, A. D., Krumpe, M., Hambaryan, V., \& Schwarz, R. 2003, A\&A, 406, 253

Stellingwerf, R. F. 1978, ApJ, 224, 953

Szkody, P., Anderson, S. F., Agüeros, M., et al. 2002, AJ, 123, 430

Warner, B. 1986, MNRAS, 219, 347

Wei, J. Y., Xu, D. W., Dong, X. Y., \& Hu, J. Y. 1999, A\&AS, 139, 575 